\title{
Estrategias efectivas para incrementar el consumo de la bebida láctea "Purita Mamá" en gestantes y nodrizas de la provincia de Ñuble, año 2012
}

\author{
Effective strategies to increasing the \\ consumption of the dairy drink "Purita Mamá" \\ for pregnant and lacting women from \\ the Ñuble province, year 2012
}

\begin{abstract}
Objective: To identify effective strategies to increase the consumption of the product "Purita Mama" in pregnant and lactating women from Nuble. Subjects and methods: Observational cross sectional study; two instruments were applied, the first one observing of consumption and acceptability of the product in pregnant and lactating women from a non-probabilistic sample; the second one applied to the health team showing major variations in the consumption from a non-probabilistic "intentional" sample. Results: The product was consumed by $38 \%$ of pregnant and $34.6 \%$ of lactating women; acceptability was regular in $54.6 \%$ and $48.2 \%$ of pregnant and lactating women, respectively. On the other hand, $34 \%$ of the professionals and technicians who work directly with pregnant and lactating women "did not know" the product. The main effective educational strategy implemented $(60.2 \%)$, is product tasting. Conclusion: The largest consumption of the product is seen in those establishments who implement effective instructional strategies.
\end{abstract}

Key words: Dairy drink "Purita Mama", effective educational strategies, National Program of Complementary Feeding.

\section{INTRODUCCIÓN}

La rápida transición epidemiológica ha producido una serie de modificaciones en la población chilena, una de ellas se ha traducido en un aumento de la obesidad y de las enfermedades cardiovasculares, lo que lo muestra la Encuesta Nacional de Salud - ENS (2010) (1). Por otra parte el Índice de Alimentación Saludable (2) indica que sólo 12\% de las mujeres presenta una alimentación saludable, 84\% debe realizar cambios en su alimentación y $4 \%$ presenta una alimentación no saludable. La malnutrición por exceso en gestantes y nodrizas fue 57,4\%, en el año 2011 en la región del Bio-Bio, Chile (3). Es de conocimiento general que en la mujer recae no sólo la reproducción biológica (embarazo, parto, puerperio, lactancia natural), sino también la responsabilidad social (cuidado de sus hijos) de la familia (4).

La población chilena presenta un bajo consumo de ácidos grasos omega-3, lo que también incluye a gestantes y nodrizas.
Gloria Sanchez S. (1)

Carolina Leyton P. (2)

Alex Medina G. (2)

(1) Servicio de Alimentación del Hospital Comunitario de Salud Familiar Quirihue del Servicio de Salud Ñuble. Chile. (2) Departamento de Gestión Empresarial de la Facultad de Ciencias Empresariales de la Universidad del Bio-Bio. Chillán, Chile.

Dirigir la correspondencia a: Profesora

Gloria Sanchez Sandoval Servicio de Alimentación Hospital Comunitario de Salud Familiar Quirihue Servicio de Salud Nuble. Chile. E-mail: gloriad.sanchez@redsalud.gov.cl

Este trabajo fue recibido el 10 de Enero de 2014 y aceptado para ser publicado el 10 de Mayo de 2014.

El bajo consumo de productos marinos (5), llevó a que desde el año 2003 el Ministerio de Salud de Chile (MINSAL), en conjunto con la Universidad de Chile, diseñan, desarrollan e incorporar una bebida láctea, Ilamada "Purita Mama", a cuyo producto le adicionaron ácidos grasos esenciales omega-3 docosahexaenoico (DHA) y eicosapentaenoico (EPA), con la finalidad de proporcionarles estos ácidos grasos poliinsaturados. Este producto es exclusivo para las gestantes y nodrizas que se encuentran inscritas en algún sistema de salud público chileno desde agosto de 2008. Con esta iniciativa Chile logró ser pionero en el mundo en este tipo de programas (6). Una importante investigación que contribuyó con la creación del producto fue el estudio de "Efectos de un producto lácteo enriquecido con múltiples micronutrientes y ácidos grasos omega-3 en el peso al nacer y la duración de la gestación en mujeres chilenas embarazadas", mejorando los pesos de nacimientos de los recién nacidos (7). 
Por lo descrito anteriormente el objetivo del presente estudio fue identificar las estrategias más efectivas para incrementar el consumo del producto "Purita Mama" en gestantes y nodrizas, para ser implementadas por los equipos de salud de atención primaria de la provincia de Ñuble - Chile.

\section{SUJETOS Y MÉTODOS}

El diseño del estudio es de tipo observacional, pasivo de caso y control, ya que selecciona dos grupos con diferente nivel de consumo del producto en el tiempo uno, y luego en el tiempo dos compara retrospectivamente para conocer cuáles fueron las estrategias que desarrollaron en cada uno de los grupos. El estudio fue realizado en las siguientes etapas:

Primera etapa: Para la aplicación del instrumento de aceptabilidad, se utilizó un muestreo No Probabilístico "por Cuota", donde para el tamaño de la muestra (8) se consideró el número de gestantes y nodrizas inscritas en cada establecimiento al año 2011, lo que constituyó un universo de 4.472, según datos de la Unidad de Estadística de la Dirección del Servicio de Salud Ñuble (9), obteniendo un tamaño muestral de 899 gestantes y nodrizas, correspondiente al 20\% del universo de acuerdo a los criterios defindos institucionalmente por los referentes técnicos de la Dirección del Servicio de Salud Ñuble (DSSÑ) y de la Delegación Provincial de Ñuble de la Secretaria Regional Ministerial de Salud (SEREMI). Participaron 41 establecimientos de salud de las 21 comunas pertenecientes a la provincia de Ñuble, la cual incluyó hospitales comunitarios de salud familiar, centros de salud familiar (CESFAM), centros comunitarios de salud familiar (CECOF) y postas. Aplicaron estos instrumentos las nutricionistas de cada establecimiento, las que en una reunión técnica provincial (mensual) recibieron la capacitación para su aplicación.

El instrumento utilizado para la recolección de datos fue el de "Aceptabilidad del Programa Nacional de Alimentación Complementaria (PNAC) elaborado y validado por el MINSAL y distribuido a la Delegación Provincial de Ñuble de SEREMI de Salud y a la DSSÑ para su aplicación. Este instrumento que ha sido utilizada anteriormente como instrumento de información para los PNAC. Dicho instrumento está conformado por tres partes, la primera describe los antecedentes generales del beneficiario, la segunda evalúa el volumen y frecuencia de consumo y la tercera evalúa las características organolépticas, el cual se aplicó previo consentimiento informado. La aplicación de éste instrumento permitió elaborar un ranking de establecimientos de acuerdo al consumo de sus gestantes y nodrizas, seleccionando cuatro de ellos con consumo superior (65\%) y cuatro establecimiento con consumo inferior (35\%).

La selección de los establecimientos permitió identificar y solicitar la colaboración para la aplicación del segundo instrumento a los 80 integrantes de los equipos de salud, conformados por: médico, odontólogo, enfermera, matrona, nutricionista, asistente social, psicólogo y técnicos paramédicos que trabajaban en forma directa con las gestantes y nodrizas. Se solicitó la colaboración de los referentes técnicos del Programa de la Mujer, del Niño y Niña, y del Chile Crece Contigo, de la DSSÑ y de la Delegación Provincial de Ñuble de la SEREMI de Salud, con la finalidad de conocer la experiencia en la implementación y evaluación de estrategias (10), junto con su percepción del producto.

Segunda parte: el diseño del segundo instrumento consideró doce preguntas semiabiertas, entre las variables estudiadas se encuentra, el consumo, características del producto y las estrategias implementadas.

Para proporcionar confiabilidad y validez al estudio se realizó un pilotaje en aproximadamente 10\% de la muestra (10 personas), donde al procesar los datos, se calculó el Coeficiente Alfa de Cronbach $(0,81)$, considerado aceptable. Por lo tanto se procedió a la aplicación del segundo instrumento mediante un "muestreo no probabilístico intencional", logrando entrevistar $100 \%$ del equipo de salud de los ocho establecimiento seleccionados en la primera etapa (80 integrantes de los equipos de salud de los establecimientos y referentes técnicos) (11). Tanto el pilotaje como el segundo instrumento fueron aplicados en terreno directamente por uno de los investigadores.

Para el procesamiento y análisis estadístico de los datos se codificó la información en programa Excel, creando dos bases de datos; los datos se introdujeron en el programa estadístico SPSS versión 17 para Windows, con lo cual se realizó un análisis descriptivo de variables, como lo fue la descripción de la muestra, nivel de consumo, características organolépticas, principalmente. Con los resultados del segundo instrumento se elaboraron tablas de contingencia, y correlación de las variables.

\section{RESULTADOS}

Los cuatro establecimientos con consumo del producto superior al 65\% fueron: CESFAM "Isabel Riquelme" (70,6\%) de la comuna de Chillán, CESFAM "Quiriquina" $(70,6 \%)$ de la comuna de San Ignacio, CESFAM "Santa Clara" $(68,4)$ de la comuna de Bulnes y CESFAM "Federico Puga" $(66,7)$ de la comuna Chillán Viejo. Los cuatro establecimientos con consumo inferior al 35\% de acuerdo a la recomendación del MINSAL fueron: Posta de "Ninhue" (10\%) de la comuna de Ninhue, Hospital Comunitario de Salud Familiar de Quirihue (30\%) de la comuna de Quirihue, CESFAM “Cobquecura" (33,3\%), de la comuna de Cobquecura y la Posta de El Carmen $(33,3 \%)$ de la comuna El Carmen.

De las gestantes $80,5 \%$ pertenecían al radio urbano y $18,2 \%$ al radio rural. En lo que respecta a las nodrizas $67,7 \%$ pertenecían al radio urbano, y 32,2\% al área rural. El total de la muestra estaba constituida por $75,8 \%$ de gestantes y $24,2 \%$ de nodrizas, de las cuales $56,6 \%$ presentaron exceso de peso y déficit nutricional en 5,1\%, de las nodrizas 59\%, presentaron un estado nutricional normal, pero $32,3 \%$ un exceso de peso. En cuanto a la dosis recomendada por el MINSAL (consumir una taza diaria) sólo $38,9 \%$ de las gestantes cumplía. Sólo $35 \%$ de las nodrizas cumplía la recomendación del MINSAL (consumir dos tazas diarias). Según la apreciación global del producto las gestantes y nodrizas $60,8 \%$ lo consideran "regular" y según su característica organoléptica de "sabor" 48,2\% lo consideran "malo".

En cuanto a los resultados del instrumento aplicado a los equipos de salud $66 \%$ mencionó conocer características del producto, sin embargo sólo 34\% mencionó las características correctas que posee el producto (más calcio, omega-3, baja en grasas, vitaminas y minerales). Un $73 \%$ proporcionó una respuesta incorrecta con respecto a la dosis recomendada para las gestantes y nodrizas. Quienes indicaron conocer la dosis recomendada, sólo 7\% mencionó correctamente la dosis para las gestantes y las nodrizas. En cuanto al conocimiento de las estrategias implementadas para incrementar el consumo del producto, en promedio $72 \%$ indica conocerlas, sin embargo de los integrantes del equipo quienes menos conocen dichas estrategias son las asistentes sociales (40\%) y los psicólogos (46\%).

Al identificar las estrategias más utilizadas por los establecimientos que presentan el más bajo consumo, fue $77,8 \%$ en "educación en la consulta sobre bebida láctea Purita Mamá" 
que de acuerdo al código sanitario la educación sanitaria consiste en capacitar al individuo y a los grupos sociales mediante acciones educativas, tendientes a compenetrarlos de su responsabilidad en los problemas de salud personal y de la comunidad y para estimular su participación activa en la solución de ellos (12). Por otra parte las estrategias más utilizadas por aquellos establecimientos que presentan un mayor consumo se encuentra $70 \%$ la "degustación del producto bebida láctea Purita Mamá" la que corresponde toda actividad educativa, de promoción y capacitación, realizada en forma regular en periodos de ingreso de productos alimentarios o de baja adherencia (13); y la estrategia menos utilizada por todos los establecimiento se encuentra la "difusión en radio u otro medio de comunicación" la que se define como el uso de medios masivos que favorece el impacto de las actividades directas de mensajes nutricionales, pero nunca puede substituirlas por completo (14)(15).

De los referentes técnicos $70 \%$ plantean que es necesario implementar nuevas estrategias para mejorar el consumo del producto, e indican (18\%) que una estrategia fundamental es mejorar la "educación al equipo de salud", y 16\% la estrategia "degustación a los funcionarios, gestantes y nodrizas" las que resultan efectivas para aumentar el consumo y agregan (14\%) la estrategia de "mejorar la entrega de la información".

\section{DISCUSIÓN}

Al incorporar un nuevo producto alimenticio a una población se debe considerar que la calidad de un alimento se determina por la cantidad y calidad de los nutrientes y por su calidad y seguridad sanitaria. Sin embargo la aceptación o rechazo se relaciona con la percepción subjetiva del consumidor, preferencia del color, sabor, textura, consistencia y presentación (16). Actualmente se prefiere la multifortificación de alimentos, debido a que las poblaciones en riesgo, generalmente presentan varias deficiencias de nutrientes simultáneamente. Esto ha hecho que países como Chile, Argentina, Guatemala y Colombia empleen un sólo alimento como transportador de varios micronutrientes, especialmente vitamina A, D, hierro, ácido fólico y zinc (17), denominados en Latinoamérica como los Programas de Alimentación Complementaria (PAC), dirigidos especialmente a grupos etarios con mayor vulnerabilidad.

Se han realizado estudios principalmente de aceptabilidad, por lo que expertos coinciden en que existe deficiente información respecto del impacto que estos programas han tenido a lo largo de los años, publicándose sólo estudios de efectividad en Chile y Panamá. El estudio de Panamá utilizó una cohorte retrospectiva, aplicado a más de 800 niños, donde se observó que el grupo intervenido presentó antes de la intervención un deterioro de la talla, pero después de la intervención una estabilización de ésta (18).

El cuanto al estado nutricional de las gestantes y nodrizas de la provincia de Ñuble, se observó que 20,8\% presentaba obesidad, valor que coincide con el "Informe de Situación Nutricional en el Sistema Público de Salud" realizado en los años 2007-2009 (19). En relación a las nodrizas 30\% presentaban un exceso de peso lo que preocupa al equipo de salud, ya que no todas ellas recuperarán su peso inicial de antes de la gestación, para lo cual el MINSAL ha implementado estrategias como lo son la Consejería Nutricional al $3^{\circ}$ y $6^{\circ}$ mes post parto (4).

En cuanto al nivel de consumo del producto de la gestante de acuerdo a lo recomendado por el MINSAL es una taza del producto una vez al día, sólo 38\% de las gestantes cumplía correctamente. El beneficio del producto está calculado para un mes, considerando 25 gramos para una taza (19), sin embargo la presente investigación arroja una alta variedad de respuestas sobre medidas entre "cucharaditas", "cucharas", entre otras, lo que dificulta evaluar la dosis correcta, por lo que se requiere que el equipo de salud en especial las nutricionistas, corrijan esta información, especialmente al momento de educar. Este problema también se presentó en el estudio de consumo realizado el año 2011, en el cual los resultados no permitieron precisar la dilución correcta del producto, siendo un problema actualmente no resuelto por el MINSAL (20).

Al evaluar las características organolépticas del producto, esto presenta una apreciación global en una escala del 1 al 7 donde 1 es muy mala, 4 regular y 7 excelente. El porcentaje promedio de respuesta fue $60,6 \%$ para la clasificación de "regular". Sin embargo al comparar la aceptabilidad de un producto alimentario, proporcionado a gestantes y niños realizado en Panamá, sobre la aceptabilidad del producto Nutricrema (21) presentó una buena valoración y aceptabilidad, lo que se puede explicar en el caso chileno, a la incorporación de aceites esenciales como es el omega-3, lo que ha llevado a la creencia popular de que el producto presenta "sabor a pescado".

En cuanto a la aplicación del segundo instrumento, 34\% de los profesionales y técnicos que trabajan en forma directa con las gestantes y nodrizas, no tiene conocimiento del producto, y $25 \%$ no conoce las estrategias que se realizan en su establecimiento para fomentar su consumo de este grupo se destacan los asistentes sociales y los psicólogos.

Lo que indica que las estrategias implementadas no se difunden y no incluyendo actividades de promoción al equipo de salud. En general todos los establecimientos en estudio indicaron que las estrategias más utilizadas en los establecimientos de salud son la "entrega de dípticos o trípticos con información de la bebida láctea Purita Mamá"; y según los estudios realizados en Chile sobre estrategias educativas, las más utilizadas son las menos efectivas. La otra estrategia mayormente mencionada es "degustación del producto bebida láctea Purita Mamá", la que se observa en los establecimientos que presentaron un mayor consumo del producto. Se puede entonces concluir que la degustación como intervención educativa es efectiva, lo que se debe realizar de forma programada y periódica $(12,22)$.

Al comparar las estrategias educativas alimentarias realizadas en Estados Unidos entre los años 2006-2011, se evidencia que las estrategias con mayor efectividad fueron aquellas en donde se involucra a la familia y la comunidad escolar, los padres son modelos a seguir, logrando aumento en el consumo de frutas, verduras y la actividad física (22). Por otra parte la Food and Agriculture Organization (FAO), hace referencia de esta modalidad, donde recomienda como una estrategia para educar e involucrar a la comunidad y las familias en temas de nutrición y salud (23). Un estudio realizado en Chile el año 2012, demuestra que las intervenciones audiovisuales, son preferidas por sobre el material impreso, por el hecho de utilizar dos sentidos (visual y auditivo); en este estudio los padres, profesores y expertos coincidieron, que los spots y videos cortos son mejor aceptados, a su vez recomendaron talleres de cocina $u$ otras actividades prácticas como forma de aprendizaje más significativas para niños y niñas (23).

Finalmente, se propone desarrollar un "Plan de Transferencia en Red" que considere: la educación al equipo de salud sobre el producto, ya que la aplicación del instrumento reflejó un desconocimiento de parte de los profesionales y técnicos que trabajan en forma directa con las gestantes y nodrizas, con el objeto de formar líderes de opinión informados; las degustaciones del producto en donde participen 
las gestantes, nodrizas y el equipo de salud; capacitación permanente a los equipos de salud, mediante talleres teóricoprácticos en la preparación del producto, con degustación de las recetas elaboradas; formación de agentes educadores en salud involucran a la comunidad y a la familia, tales como madres con experiencia en una situación similar y experiencia positiva con el producto, asumiendo un rol protagónico como multiplicadores de la información y experiencia. Este tipo de experiencias en EEUU ha logrado resultados positivos para la prevención de la obesidad en escolares 2006-2011, además la FAO incentiva este tipo de estrategias desde el año 1984 para mejorar la nutrición en la población. Altamente relevante resultará la evaluación y monitoreo del Plan de Transferencia en Red, el cual también se sugiere incorporarlo dentro de las estrategias del programa Chile Crece Contigo.

\section{CONCLUSIONES}

El producto presenta un bajo nivel de consumo; y sólo $38 \%$ de las gestantes y $34,6 \%$ de las nodrizas cumplen con la dosis recomendada por el MINSAL. La apreciación global de producto es "regular", en donde se destaca que la principal causa de rechazo es el sabor y olor.

En los establecimientos en los cuales existe un bajo consumo del producto, la estrategia "educación en la consulta sobre bebida láctea Purita Mamá" se realizaba con mayor frecuencia por sobre las otras estrategias. En los establecimientos en donde se presenta un mayor consumo de la "Purita Mama", existen estrategias que involucran mayormente a la familia y comunidad, como son las degustaciones.

El producto bebida láctea "Purita Mama" requiere de estrategias educativas efectivas, donde se destaquen sus beneficios y que aumente su correcto consumo en la población objetivo.

Resulta necesario reenfocar el trabajo de los profesionales bajo el Modelo de Salud Familiar ya que se destacan las respuestas "mayor involucramiento del equipo de salud", o "trabajo en equipo".

Finalmente, es necesario diseñar estrategias innovadoras para fomentar el consumo del producto, implementando un Plan de Transferencia en Red, diseñado de acuerdo a las necesidades de los equipos de salud, referentes técnicos de los Servicios de salud y de las SEREMI de Salud y por sobre todo de la Población objetivo.

\section{RESUMEN}

Objetivo: identificar estrategias efectivas para que los equipos de salud de la provincia de Nuble - Chile incrementen el consumo del producto lácteo "Purita Mama" para gestantes y nodrizas. Sujetos y métodos: estudio observacional, pasivo de caso y control donde se aplicaron dos instrumentos, el primero de consumo y aceptabilidad del producto en gestantes y nodrizas a una muestra no probabilística "por cuota"; y el segundo, aplicado a los equipo de salud que presentó mayores variaciones en el consumo a una muestra no probabilística "intencional". Resultados: Consumen el producto 38\% de las gestantes y $34,6 \%$ de las nodrizas; de las que lo consumen su aceptabilidad es regular en $54,6 \%$ de las gestantes y en $48,2 \%$ de las nodrizas. Por otra parte "no conocen el producto" 34\% de los profesionales y técnicos que trabajan directamente con gestantes y nodrizas. La principal estrategia educativa efectiva implementada (60.2\%), es la "degustación del producto". Conclusión: El mayor consumo del producto, se presenta en aquellos establecimientos donde se implementan estrategias educativas efectivas.
Palabras clave: Bebida láctea "Purita Mama", estrategias educativas efectivas, Programa Nacional de Alimentación Complementario.

Agradecimientos: Para los profesionales Nutricionista de la Red Asistencial de la Provincia de Ñuble. Para el Departamento de Red Asistencial del Servicio Salud Ñuble. Para los docentes del Magíster en Salud Pública de la Universidad del Bio-Bio.

Fuente de financiamiento: La investigación fue financiada con recursos propios de los investigados, por lo que no presenta conflictos de interés.

\section{BIBLIOGRAFÍA}

1. Ministerio de Salud Chile. Resultados de la Encuesta Nacional de Salud 2009-2010. MINSAL 2010. [En Línea] Disponible en: <http://www.minsal.cl/portal /url/item/ bcb03d7bc28b64dfe040010165012d23.pdf. [Citado el: 21 ene 2013].

2. Pereyra I., Erazo M, Evaluación de la alimentación en mujeres atendidas por el Sistema de Salud Público Chileno. Rev. ALAN. [En Línea] Dic 2011; 61 (3) Disponible en : <http://www.alanrevista.org/ediciones/2011/3/?i=art4> [Citado el: 14 dic 2012].

3. Departmento de Estadística e Informaciónde Salud. Indicadores Basicos de Salud, 2011. [En Línea] Ministerio de Salud. Disponible en: <http://deis.minsal.cl /deis/indicadores/Folleto_IBS_2011.pdf > [Citado el: 20 ago 2012]

4. Pizarro T. Intervención nutricional a través del ciclo vital para la prevención de obesidad y otras enfermedades crónicas no transmisibles. Rev Chil Pediatr. [En Línea]. Feb 2007; 78(1): 76-83. Disponible en : <http://www. scielo.cl /scielo.php?script=sci_arttext\&pid $=$ s037041062007000100011\&/ng=es. Http://dx.doi.org/ 10.4067/s0370-41062007000100011> [Citado el: 14 jun 2013].

5. Atalah E., Vera G., Rosselot G., Araya H., Andreu R., Alviña M., et al. Desarrollo, consumo y aceptabilidad de una bebida láctea con DHA para embarazadas y Nodrizas. [En línea]. Rev Chil Nutr. Dic 2008; 35 (4). Disponible en:<http:// www.scielo.cl/scielo.php?script=sci_arttext\&pid=S071775182008000500006\&lng=es>. [Citado el: 27 jul 2012.]

6. Ministerio de Salud, Unidad de Alimentos y Nutrición. Estrategia de Intervención Nutricional a través del Ciclo Vital para la Prevención de Obesidad y otras Enfermedades No Transmisibles. [En línea] Mayo 2010. Disponible en: <http://web.minsal.cl/portal/url/item/8a2cacad32ce5b 9ce04001011e017fb4.pdf> [Citado el: 30 ago 2012.]

7. Mardones F, Urrutia MT, Villarroel L, Rioseco A, Castillo $O$, Rozowski J, Tapia JL y cols. Effects of a dairy product fortified with multiple micronutrients and omega-3 fatty acids on birth weight and gestation duration in pregnant Chilean women. [En línea] Public Health Nutr 2008; 11: 30-40. Disponible en: http://www.ncbi.nlm.nih.gov/ pubmed/17565762 [Citado el: 8 mayo 2014.]

8. Pineda B, De Alvarado E, Hernández F. Metodología de la investigación, manual para el desarrollo de personal de salud. Washinngton DC: OPS. 1994.

9. Servicio de Salud Ñuble, Subdirección Médica, Departamento de Bioestadística e Información de Salud. Población total por establecimiento y comuna, 2011.

10. Huerta Riveros Patricia C, Paúl Espinoza Iván R, Leyton Pavez Carolina E. Impacto de indicadores de gestión 
en salud sobre estrategias de un servicio de salud público. Rev. Salud Pública [serial on the Internet]. 2012 Apr; 14(2): 248-259. Available from: http://www. scielo.org.co/scielo.php?script=sci_arttext\&pid=S012400642012000200006\&lng=en. [Citado el: 8 mayo 2014.]

11. Foltz J, Mayo A, Belay B, Nihiser A, Dooyema C, Blanck H. Population-Level Inervention Strategies and Examples for Obesity Prevention in Children. [En Línea] Ann Rev Nutr. August 2012; 32: 391-415, Disponible en: <http:// www.annualreviews.org/doi/full/10.1146/annurevnutr-071811-150646>, [Citado el: 15 mayo 2013].

12. Ministerio de Salud Chile. Código Sanitario Decreto Con Fuerza de Ley $N^{\circ}$ 725, 31 de enero 1968, [En Línea] Disponible en: http://www.leychile.cl/Consulta/m/norma_ plana?idNorma=5595\&org=cdr [Citado el: 12 ago de 2012]

13. Rodríguez L, Barba C, Contreras A, Benavides X, Cornejo J. Manual de Programas Alimentarios. MINSAL. Santiago. 2011: 7 -19.

14. Riquelme $H$ Giselle, Concha P Ximena, Urrutia S María Teresa. Intervenciones educativas para la prevención del cáncer cervicouterino. Rev Chil Obstet Ginecol. [revista en la Internet]. 2012; 77(2): 111-115. Disponible en: http://www.scielo.cl/scielo.php?script=sci_arttext\&pid =S0717-75262012000200006 [Citado el: 15 abril 2013]

15. Puldón $V$, Suárez $E$, Caraballo RA, Pachón H, Martínez CP. Preferencia y aceptabilidad de la variedad de arroz IACuba 30 con alto contenido de hierro y zinc por mujeres gestantes en Cuba. [En línea] Perspect Nutr Humana. 2011;13:123134. Disponible en: <http://www.scielo.org.co/pdf/penh/ v13n2/v13n2a2> [Citado el: 15 nov 2012].

16. López BE, Carvajal LM. Elaboración de un alimento con base en harina de banano (Musa paradisiaca) fortificada con hierro y zinc aminoquelados, calcio microencapsulado y folato. [En línea] Perspect Nutr Humana. 2012;14: 4757. Disponible en: < http://revinut.udea.edu.co/index.
php/nutricion/article/viewFile/10365/12433> [Citado el: 26 abril 2013].

17. Galván M, Amigo H. Programas destinados a disminuir la desnutrición crónica, una revision para america latina. [En línea] Rev ALAN, dic 2007; 57 (4). Disponible en : <http:// WWw.alanrevista.org/ediciones/2007/4/?i=art2. > [Citado el: 20 jul 2012).

18. Ministerio de Salud, Proteccion de la Salud. Estrategia de intervención nutricional a través del ciclo vital. Situación nutrición en el sistema público de salud 2007-2009. [En línea] 2012 Disponible en: http://www.minsal.gob.cl/ portal/url/tem/89185968d325a51ee04001011e010c92. pdf> [Citado el: 12 mayo 2013].

19. Contreras A, Herrera Y, Rodríguez L, Pizarro T, Atalah E. Aceptabilidad y consumo de una bebida láctea con omega-3 en embarazadas y Nodrizas del programa nacional de alimentación complementaria. Rev Chil Nutr. 2011; 38 (3): 313-20.

20. De Caballero E, Atalah E. Evaluación de la aceptabilidad y consumo de un suplemento alimentario en la República de Panamá. Rev Chil Nutr. 2003; 30: 2.

21. Foltz J, Mayo A, Belay B, Nihiser A, Dooyema C, Blanck $H$. Population-Level Inervention Strategies and Examples for Obesity Prevention in Children. [En Línea] Ann Review Nutr. 2012; 32: 391-415, Disponible en: <http:// www.annualreviews.org/doi/full/10.1146/annurevnutr-071811-150646>, [Citado el: 15 mayo 2013].

22. FAO. Manual, "Selección de intervenciones para mejorar la nutrición". Roma: s.n., 1984: 4-18.

23. Vio F, Lera L, Fuentes-García A, Salinas J. Método Delphi para identificar materiales educativos sobre alimentación saludable para educadores, escolares y sus padres. [En línea] Rev ALAN. 2012; 62 (3). Disponible en: <http:// www.alanrevista.org/ediciones/2007/4/?i=art2. > [Citado el: 20 mayo 2013). 